\title{
Involvement of XZFP36L1, an RNA-binding protein, in Xenopus neural development
}

\author{
Yingjie XIA ${ }^{1,2, \#}$, Shuhua $\mathrm{ZHAO}^{3, \#}$, Bingyu $\mathrm{MAO}^{1, *}$ \\ 1. State Key Laboratory of Genetic Resources and Evolution, Kunming Institute of Zoology, the Chinese Academy of Sciences, Kunming 650223, China \\ 2. Graduate University of Chinese Academy of Sciences, Beijing 100049, China \\ 3. Yunnan Key Laboratory of Fertility Regulation and Minority Eugenics, Yunnan Population and Family Planning Research Institute, Kunming 650021, China
}

\begin{abstract}
Xenopus ZFP36L1 (zinc finger protein 36, C3H type-like 1) belongs to the ZFP36 family of RNA-binding proteins, which contains two characteristic tandem CCCH-type zinc-finger domains. The ZFP36 proteins can bind AU-rich elements in 3' untranslated regions of target mRNAs and promote their turnover. However, the expression and role of ZFP36 genes during neural development in Xenopus embryos remains largely unknown. The present study showed that Xenopus ZFP36L1 was expressed at the dorsal part of the forebrain, forebrain-midbrain boundary, and midbrain-hindbrain boundary from late neurula stages to tadpole stages of embryonic development. Overexpression of XZFP36L1 in Xenopus embryos inhibited neural induction and differentiation, leading to severe neural tube defects. The function of XZP36L1 requires both its zinc finger and $\mathrm{C}$ terminal domains, which also affect its subcellular localization. These results suggest that XZFP36L1 is likely involved in neural development in Xenopus and might play an important role in post-transcriptional regulation.
\end{abstract}

Keywords: ZFP36L1; RNA-binding protein; Neural development; Xenopus; Post-transcriptional regulation

mRNA stability is an important mechanism for gene expression regulation, which is critical for embryogenesis and normal physiological processes. The zinc finger protein 36 (ZFP36) family proteins are key players in post-transcriptional regulation and contain two or more highly conserved tandem $\mathrm{CCCH}$ zinc fingers (TZF) of tandem $\mathrm{Cx} 8 \mathrm{Cx} 5 \mathrm{Cx} 3 \mathrm{H}$ repeats ( $\mathrm{x}$ represents a variable amino acid). These factors are able to bind to AU-rich elements (AREs) in the 3' untranslated regions (UTR) of targeted mRNAs through their TZFs (Carballo et al, 2000; Lai \& Blackshear, 2001; Lai et al, 1999; Lai et al, 2000), which leads to the removal of the poly(A) tail off the targeted mRNAs and promotes their degradation (Carrick et al, 2004; Lai \& Blackshear, 2001). The ZFP36 family proteins are evolutionarily conserved, including four rodent members (ZFP36, ZFP36L1, ZFP36L2, and ZFP36L3) and three mammal members (with ZFP36L3 missing, Blackshear et al, 2005), each of which has two conserved ZF domains. Four members have been reported in Xenopus and zebrafish, among which ZFP36, ZFP36L1, and ZFP36L2 are conserved while the fourth member (Xenopus ZFP36L2.1, ZFP36L2.2 and zebrafish ZFCTH1) contains four ZF domains instead of two. The four ZFP36 genes in Xenopus have been cloned and are widely expressed in adult tissues, except for XZFP36L2.1 (XC3H-4), which is detected only in the ovary (De et al, 1999). It has been suggested that XZFP36L2b (XC3H-3b) plays an important role in Xenopus kidney development (Kaneko et al, 2003), but little is known about the roles of other ZFP36 members in embryonic development.

Also known as cMG1, TIS11b, ERF1, BRF1, C3H2, and Berg36 (Barnard et al, 1993; De et al, 1999; Lai et al, 1990; Varnum et al, 1991), ZFP36L1 has been reported to regulate vascular endothelial growth factor (VEGF) mRNA stability in adrenocorticotropic hormone-stimulated primary adrenocortical cells acting as a negative regulator of VEGF during development

Received: 17 August 2012; Accepted: 15 October 2012

Foundation items: This work was supported by National Natural Science Foundation of China (90919039; C120106)

\# These authors contributed equally to this work

* Corresponding author, E-mail: mao@mail.kiz.ac.cn 
(Ciais et al, 2004). Research has also shown ZFP36L1 to be regulated by Von Hippel-Lindau (VHL) tumor suppressor protein in renal cancer cells (Sinha et al, 2009). In addition, ZFP36L1 is phosphorylated by p38 MAPK/MAPK-activated protein kinase 2 (MK2) and PKB/AKT pathways. Phosphorylation of ZFP36L1 inhibits its ARE mRNA decay activity, but does not affect its ability to bind AREs (Benjamin et al, 2006; Maitra et al, 2008; Schmidlin et al, 2004). Due to a failure of chorioallantoic fusion, ZFP36L1 knockout mice died in utero before embryonic day 11 (E11) (Stumpo et al, 2004). The XZFP36L1 gene contains 345 amino acids and shows $76 \%$ identity to human BRF-1 (De et al, 1999). However, the developmental expression and function of Xenopus ZFP36L1 remains largely unknown. In this study, we cloned XZFP36L1 and investigated its expression pattern and potential function during Xenopus laevis embryo development.

\section{MATERIALS AND METHODS}

\section{Plasmid construction}

The XZFP36L1 full open reading frame (ORF) and all truncated fragments were amplified by polymerase chain reaction (PCR) using the XL073b24 clone as a template, which was provided to us by the National Institute for Basic Biology, Japan (NIBB) The PCR primers were:

XZFP36L1 ORF: forward: 5'-ATGTCTACAGCTTTGAT TTCTCC-3'

and reverse: 5'- ATCATCAGAGATAGAAAGTCTGC-3'; N-term truncate: forward: 5'-ATGTCTACAGCTTTGAT TTCTCC-3'

and reverse: 5'-CTGTCCCCCAGGCTTTTGGAGAA-3'; NZF truncate: forward: 5'-ATGTCTACAGCTTTGAT TTCTCC-3'

and reverse: 5'- TCTCTCTTCTGCATTGTGGATG-3'; ZF domains: forward: 5'-CAGGTGAACTCCAGCAGG TACAAG-3'

and reverse: 5'- TCTCTCTTCTGCATTGTGGATG-3'; ZFC truncate: forward: 5'- CAGGTGAACTCCAGCAG GTACAAG -3'

and reverse: 5'- ATCATCAGAGATAGAAAGTCTGC-3'; C-term truncate: forward: 5'-GAGAGACGCTTGGTAT CAGGAC-3'

and reverse: 5'- ATCATCAGAGATAGAAAGTCTGC-3'.

For expression in Xenopus embryos and cultured cells, the fragments were cloned into a eukaryotic expression vector pCS2+ with a Flag tag coding sequence at the $\mathrm{C}$-terminal.

\section{Phylogenetic analysis of the ZFP36 protein family}

Twenty full coding amino acid sequences of the ZFP36 family were used for Bayesian phylogenetic analysis, including ZFP36_H.sapi (AAH09693),
ZFP36_M.musc (AAH21391), ZFP36_X.laev (NP_001081884), ZFP36_D.rerio (XP_002665468), ZFP36_C.elegans (NP_505927), ZFP36̄L1_H.sapi (NP_004917), ZFP36L_1_ M.musc (NP_031590), ZFP36L1A_X.laev (NP_001089645), ZFP36L1B_X.laev (NP_001084214) ZFP36̄L1A_D.rerio (NP_001070621), ZFP36L1B_D.rerio (NP_955943), ZFP36L2_H.sapi (NP_008818), ZFP36L2_M.musc (NP_001001806), ZFP36L2A X.laev (NP 001080610), ZFP36L2B X.laev (NP_001081886), ZFP36L2_D.rerio (NP_996938), ZFP36L2.1_X.laev (NP_001081888), ZFP36L2.2_X.laev (NP_001108269), ZFP36L3_M.musc (NP_001009549), and ZFCTH1_D.rerio (NP_571014).

Caenorhabditis elegans ZFP36 (C3H-1) were included as an out-group. Sequence alignment was performed using Clustal W (Thompson et al, 1994). The Bayesian approach was implemented in MrBayes3.12 (Ronquist \& Huelsenbeck, 2003) with estimation of the substitution rate model, and gamma distribution of among site rate variation two runs were used, each with a single chain of $2 \times 10^{7}$ generations, sampled every 10000 generations. Convergence was assessed by comparing the standard deviation of split frequencies between runs. We excluded 1000 trees from a total sample of 2001 trees in each run. Sample independence was confirmed as no significant increase in log-likelihoods observed after the burn-in phase.

\section{In situ hybridization, section and RT-PCR analysis}

Whole mount in situ hybridization (WMISH) on $X$. laevis was carried out as previously described (Gawantka et al, 1995). To examine potential overlapping expression, digoxigenin- and fluorescein-labeled RNA probes were used for double WMISH. Plasmid XL073b24 was cut using BamHI, and transcribed with T7 RNA polymerase to generate an antisense probe of XZFP36L1. The probes of X. laevis N-Tubulin (Papalopulu \& Kintner, 1996), Sox2 (Ma et al, 2007), Slug (Aybar et al, 2003), Xath1 (Kim et al, 1997), En2 (Hemmati-Brivanlou et al, 1991), Pax3 (Sato et al, 2005), Dbx2 (Ma et al, 2011), and Nkx6.2 (Zhao et al, 2007) were used as previously described. Stained embryos were embedded in paraffin, sectioned at $20 \mu \mathrm{m}$, and photographed using a light microscope (Leica).

Semi-quantitative reverse transcription PCR was carried out as previously described (Wu et al, 2010). The PCR primers and conditions were:

XZFP36L1A: forward: 5'-TGCATGAGCAGCAGACGT G-3' and reverse: 5'-GGTCTTATGCGTCTCTCCATG-3', 30 cycles;

XZFP36L1B: forward: 5'-TGCATGAGCAGCAGAGGA A-3' and reverse: 5'-GCCCTTCTGTGTCTCTAAACA3', 30 cycles; $H 4$ was used as a loading control: forward: 5'-CGGGA TAACATTCAGGGTA-3' and reverse: 5'TCCATGGCG GTAACTGTC-3', 26 cycles. 


\section{Embryo culture and microinjection}

In vitro fertilization, embryo culture, and WMISH of Xenopus embryos were carried out as per Gawantka et al (1995). Embryos were staged according to Nieuwkoop and Faber (1967). Capped mRNA of X. laevis ZFP36L1 and its truncated forms were synthesized in vitro using SP6 mMessage Machine kit (Ambion). Microinjection was carried out using the PLI-1 Pico-injector (Harvard Apparatus) equipped with an MK-1 micromanipulator (Singer Instruments). Embryos were injected in one dorsal blastomere at the 4-cell stage with $0.5-1 \mathrm{ng}$ XZFP36L1 mRNA or 0.3-0.6 ng of truncated mRNA. Injected mRNA was traced by co-injected $100 \mathrm{pg}$ nuclear $\beta$-galactosidase, which was stained with Red-Gal substrate (Research Organics). The detection of $\beta$ galactosidase activity for tracing cell lineage was carried out as previously described (Kurata \& Ueno, 2003).

\section{Cell transfection and immunohistochemistry}

The XZFP36L1 ORF and its truncated constructs were cloned in frame into a eukaryotic expression vector pCS2+ with a C-terminal Flag tag coding sequence. Hela cell culture, transfection, and immunohistochemistry were carried out as described in previous research (Wu et al, 2010).

\section{RESULTS}

\section{Cloning and phylogenetic analysis of ZFP36 family} proteins

Through BLAST search against EST databases, two alleles of $X$. laevis ZFP36L1 were found, XZFP36L1A and XZFP36L1B, which shared $97 \%$ identity at the amino acid level. The full ORF of XZFP36L1B was cloned by PCR using an EST clone as a template (XL073b24, from the National Institute for Basic Biology, Japan). In the following studies on XZFP36L, this allele was used unless otherwise stated. Consistent with its mammal orthologues, XZFP36L1 had two tandem ZF domains. To confirm the evolutionary relationships between the homologues of ZFP36L1, a phylogenetic tree was constructed using MrBayes. The nomenclature of the XZFP36 members was according to Xenbase (Bowes et al, 2008). The ZFP36 family proteins were grouped into four well-supported vertebrate clades inferred from the phylogenetic results (Figure 1). The results indicate that ZFP361, ZFP36L1, ZFP36L2, and ZFP36L3 were indeed paralogues that arose by gene duplication from a common ancestor to the vertebrate lineage. However, ZFP36L3 in rodents and the fourth ZFP36 genes in fish (ZFCTH1) and frog (ZFP36L2.1 and 2.2) were lineage specific. Among the ZFP36 related proteins, members of the ZFP36L1 and ZFP36L2 subfamilies were more closely related.

Temporal and spatial expression of XZFP36L1 during Xenopus laevis early development

We examined XZFP36L1 expression during $X$. laevis embryogenesis by RT-PCR and WMISH. Both XZFP36L1A and B showed similar temporal expression patterns, as detected by RT-PCR. They were both expressed at low levels maternally and in embryos from blastula to gastrula stages. Their expression went up at early neurula stages and remained stable at later stages (Figure 2A).

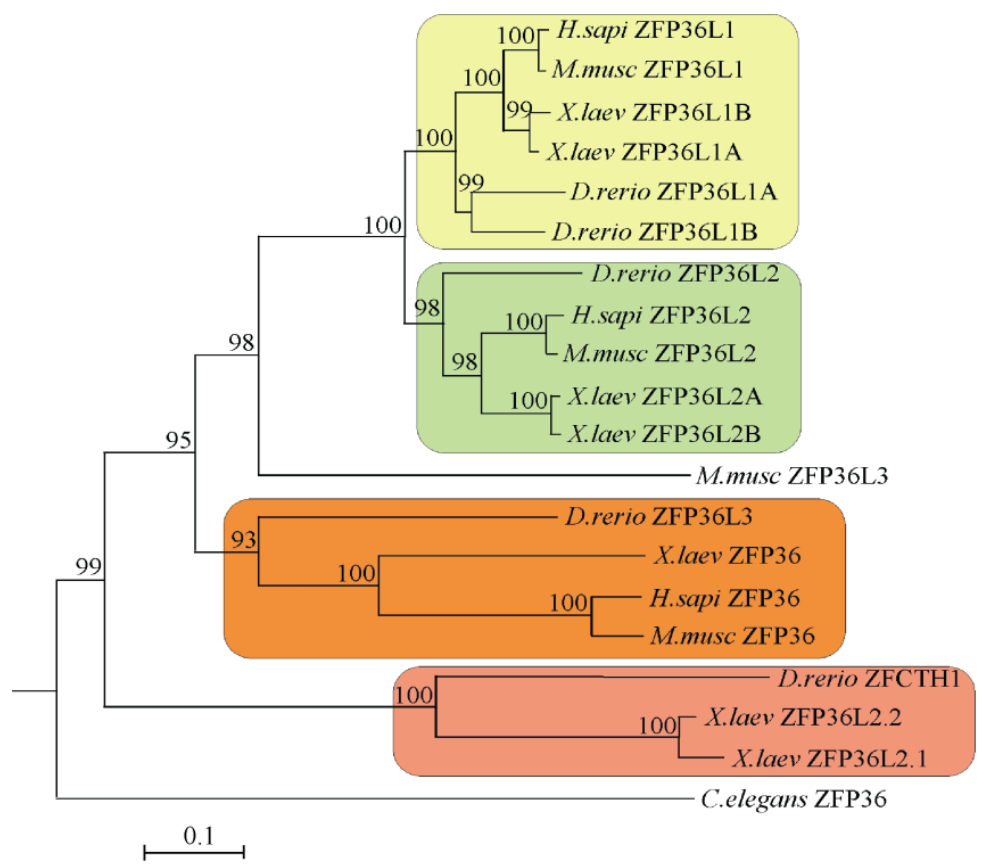

Figure 1 Bayesian tree depicting the phylogenetic relationships of the ZFP36 proteins. Branch confidence values are shown as Bayesian posterior probabilities. The scale bar shows the number of substitutions per site. 

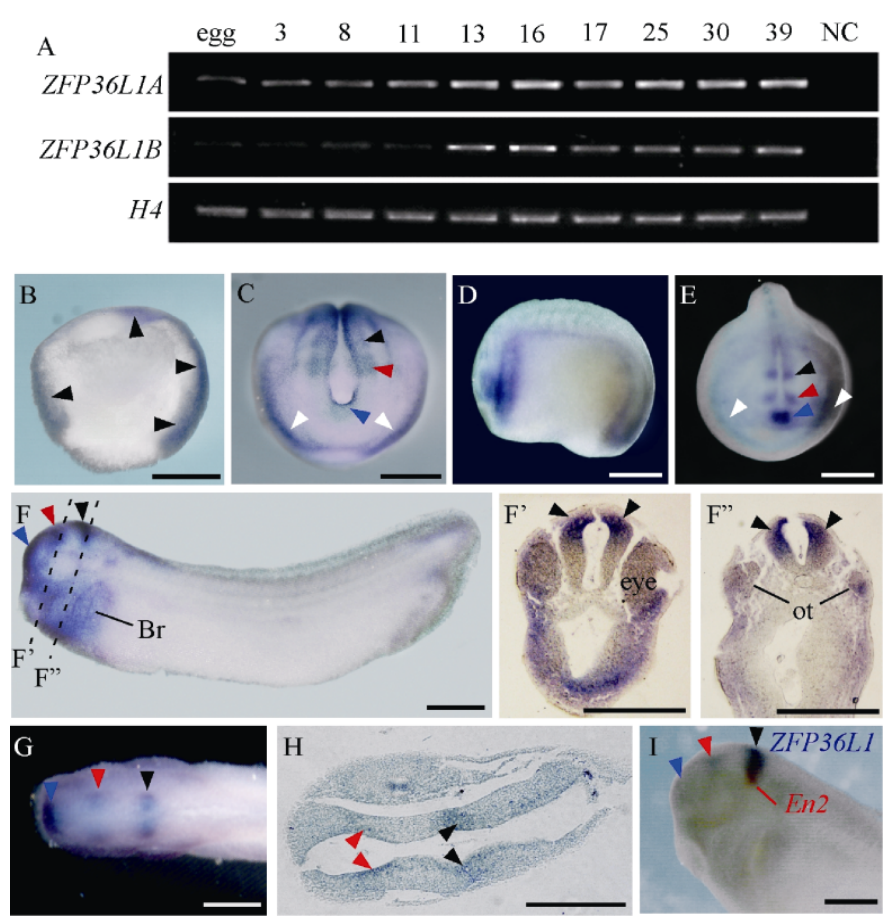

Figure 2 Temporal and spatial expression patterns of XZFP36L1

A: RT-PCR analysis of the developmental expression of XZFP36L1A and B; B-I: Expression patterns of XZFP36L1 revealed by WMISH and paraffin sections; B: Stage 10, the embryo was bisected, the arrowheads indicate the expression of XZFP36L1 in the ectoderm and involuting mesoderm; C: Stage 16, anterior view; D: Stage 20, lateral view; E: Stage 20, anterior view; F-I: Stage 32 embryos: F: Lateral view. The broken lines indicate the position of corresponding sections showed in F' and F". Br, branchial arches. F', F": Transverse sections of stage 32 embryo, the arrowheads indicate the expression of XZFP36L1 in the dorsal brain. ot, otic vesicle; G: Dorsal view of the head region; H: Horizontal section showing its expression in the fore-midbrain boundary (red arrowheads) and mid-hindbrain boundary (black arrowheads); I: Double WMISH of En2 (red) and XZFP36L1 (blue). Arrowheads in (C-I) indicate the expression of XZFP36L1 in the forebrain (blue), fore-midbrain boundary (red) and mid-hindbrain boundary (black). White arrowheads in (C) and (E) indicate its expression at the presumptive neural placode region. Bars in $(B-I)=0.5 \mathrm{~mm}$.

Since the two alleles of XZFP36L1 shared more than $90 \%$ identity, it was difficult to design probes to distinguish their expression patterns by in situ hybridization. Here we used XZFP36L1B as a template for in situ probe. At the gastrula stage, XZFP36L1 transcripts were detected in both ectoderm and mesoderm (Figure 2B). During neurula stages, its expression became enriched in the neural system, especially in the anterior neural folds (Figure 2C), where their expression became gradually restricted into three discontinuous patches, corresponding to the presumptive forebrain, fore-midbrain boundary. and mid-hindbrain boundary, respectively (Figure 2D, E). Its expression was also detected in the placode regions at the anterior neural plate borders (Figure 2C, E). At tadpole stages, XZFP36L1 remained expressed in the three discontinuous regions of the brain and also in the branchial arches (Figure 2F, G). These domains were confirmed to be the forebrain, fore-midbrain boundary, and mid-hindbrain boundary, respectively, by horizontal sections (Figure 2H, arrow heads) and double in situ hybridization with En2, a mid-hindbrain boundary marker (Figure 2I). Transverse sections of stage32 embryos showed that XZFP36L1 was mainly expressed at the dorsal part of the neural tube (Figure 2F', F"). These data showed that XZFP36L1 was expressed in specific brain regions during neurulation and might play a role in neural development.

\section{Overexpression of XZFP36L1 leads to neural tube defects}

To investigate the function of XZFP36L1 during Xenopus embryogenesis, we overexpressed XZFP36L1 in Xenopus embryos by injecting synthetic XZFP36L1 mRNA into one dorsal cell at the 4-cell stage. Interestingly, the injected embryos showed severe neural tube defects (NTDs). Compared to the control group, the neural tubes of the injected embryos failed to close properly and remained open at tailbud stages $(57 \%, n=81$, Figure $3 \mathrm{~A}, \mathrm{~B})$. To further investigate the role of XZFP36L1 in neural development, we checked the expression of several neural markers in ZFP36L1 mRNA injected embryos. The results showed that many of the neural markers were inhibited at neurula and tailbud stages, including $N$-tubulin $(67 \%, n=24$, Figure $3 \mathrm{C})$, Sox2 $(86 \%, n=35$, Figure 3D), Slug $(85 \%, n=20$, Figure $3 \mathrm{E}), \operatorname{En} 2(84 \%, n=25$, Figure 3F), $N k x 6.2(73 \%, n=15$, Figure 3G), $\operatorname{Pax} 3(70 \%, \mathrm{n}=17$, Figure $3 \mathrm{H}), \operatorname{Dbx} 1$ (69\%, $\mathrm{n}=16$, Figure 3I), and Xath1 $(62 \%, n=13$, Figure 3J).

Volume 33 Issues E5-6 


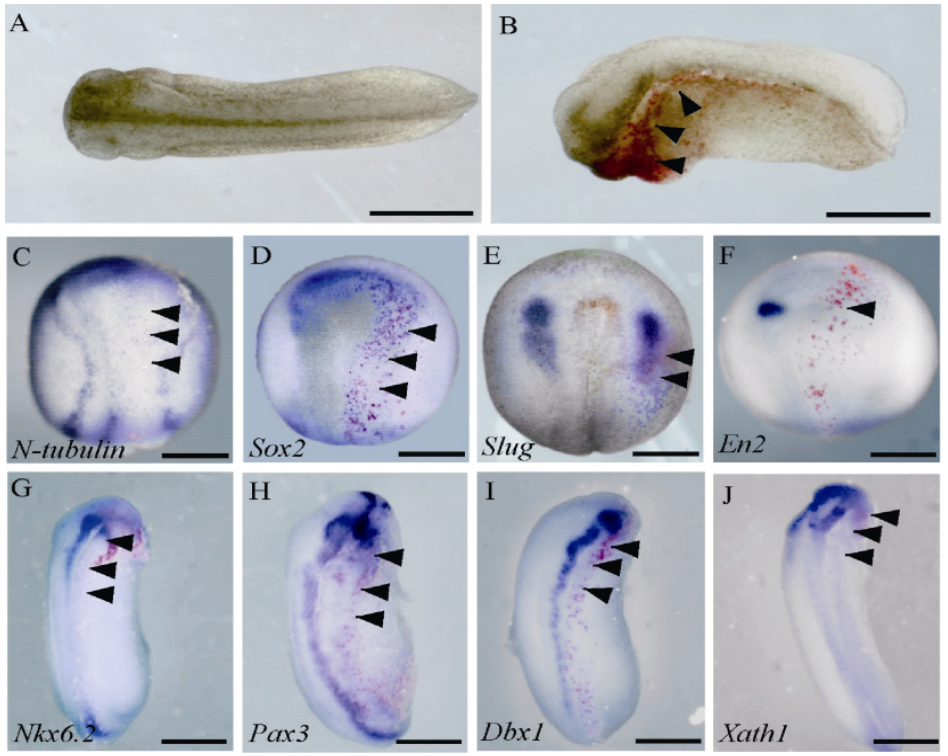

Figure 3 Overexpression of XZFP36L1 leads to NTDs in Xenopus embryos

A: Control embryos, stage25; B: Embryo injected with XZFP36L1 mRNA shows severe defects during neural tube closure (arrowheads), stage 25; C-J: Overexpression of XZFP36L1 inhibits neural differentiation. Expression of neural marker genes N-tubulin (C), Sox2 (D), Slug (E), En2 (F), Nkx6.2 (G), Pax3 (H), Dbxl (I) and Xathl (G) is largely inhibited in the injected sides (arrowheads). (C-F), stage 15; (G-J), stage 25. Bars=0.5 mm.

These results suggested that overexpression of XZFP36L1 inhibited neural induction and differentiation in Xenopus embryos, leading to severe neural tube defects.

The ZF and C-terminal domains of XZFP36L1 are required for its activity and subcellular localization

The XZFP36L1 protein contains an N-terminal domain, two ZF domains, and a C-terminal domain (Figure 4A). To determine which domains are essential for its function in neural development, several truncated forms of XZFP36L1 were constructed (Figure 4A), transcribed into mRNAs, and injected into Xenopus embryos. Embryos were examined at stage 20, when the neural tube was completely closed. The results showed that truncates containing both the ZF and the C-terminal domains led to defects in neural tube closure for Xenopus embryos like the full length ORF (Figure 4F), whereas other truncated forms lacking either the ZF motif or the C-terminal domain did not exhibit such an effect (Figure $4 \mathrm{D}, \mathrm{E}, \mathrm{G})$. These results suggest that both the ZF and the $\mathrm{C}$-terminal domains were necessary for its function.

The XZFP36L1 gene belongs to the ZFP36 RNAbinding protein family. The ZF domains of ZFP36 family proteins are suggested to bind to the AREs of targeted 3' UTRs. Thus, these proteins are supposed to locate in the cytoplasm to execute their functions. To determine whether the activity of XZFP36L1 in neural development was related to its subcellular localization, we fused the full-length XZFP36L1 ORF and its truncates with a Cterminal Flag tag coding sequence and performed immunohistochemistry using anti-Flag antibodies in transfected Hela cells. As expected, the full length ORF was detected dominantly in the cytoplasm, which was consistent with human ZFP36L1 (CMG1) results (Figure $4 \mathrm{H}$; Phillips et al, 2002). The construct containing only the N-terminal domain showed granular distribution in the cytoplasm (Figure 4I). All the other truncated forms were more enriched in the nucleus, except for the one containing both the ZF and C-terminal domains, which showed higher signal in the cytoplasm compared to other truncates (Figure 4J-M). According to the above data, the activity of these truncates in NTDs seemed consistent with their localization in the cytoplasm, indicating that the cytoplasmic localization of ZFP36L1 was important for its function.

\section{DISCUSSION}

In this study, we showed that XZFP36L1 was expressed in specific brain regions in Xenopus embryos, and overexpression of this protein inhibited neural induction and differentiation and led to severe NTDs, indicating that XZFP36L1 was involved in Xenopus neural development. We also tried to knockdown the expression of endogenous ZFP36L1 using specific morpholinos. Unfortunately, no clear phenotypes were observed in the injected embryos, although the morpholinos actively inhibited the expression of the reporter GFP genes carrying the targeted sequences (data not shown). The reason for this might be that either the morpholino was not efficient enough to block XZFP36L1 expression, or its function was compensated by other members of this gene family, since it has been 

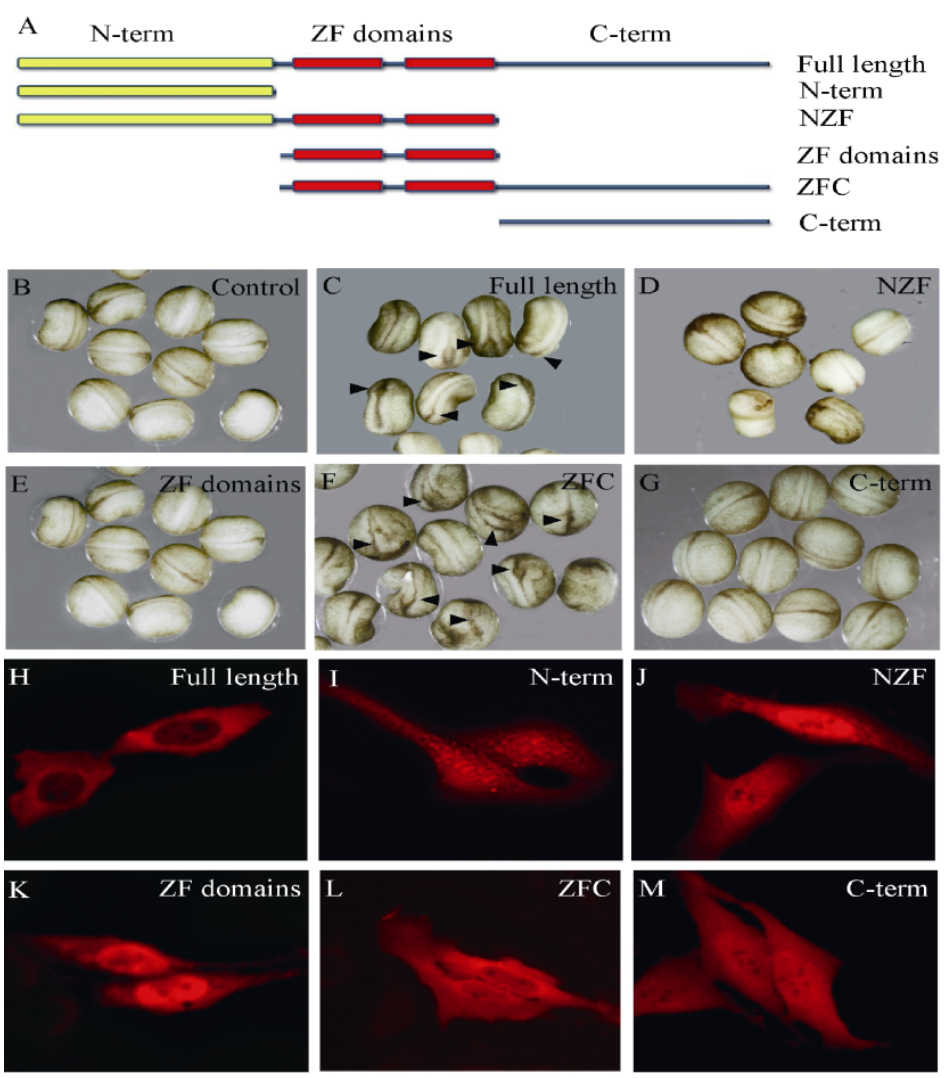

Figure 4 Activity and cellular localization of different XZFP36L1 truncates

A: The schematic structures of XZFP36L1 protein and its truncates; B-G: Both the ZF motif and the C-terminal domain are required for its activity in neural development; B: control embryos. Overexpression of full length XZFP36L1 (C) or the ZFC truncate (F) leads to defects in neural tube closure (arrowheads), while overexpression of the NZF truncate (D) or ZF domains (E) or C-terminal truncate (G) has no obvious effect on neural tube closure; H-M: Localization of XZFP36L1 and its truncates in transfected Hela cells.

reported that the ZFP36 protein family are all RNAbinding proteins and may share some common target mRNAs, e.g., TNF- a (Carballo et al, 1998; Lai et al, 2000), GM-CSF(Carballo et al, 2000; Lai \& Blackshear, 2001), and VEGF(Bell et al, 2006; Brennan et al, 2009; Ciais et al, 2004).

The function of XZP36L1 required both its ZF and $\mathrm{C}$ terminal domains, the $\mathrm{ZF}$ domains for binding to AREs and targeting mRNA degradation and the C-terminal domain to contain nucleus export sequences responsible

\section{References}

Aybar MJ, Nieto MA, Mayor R. 2003. Snail precedes slug in the genetic cascade required for the specification and migration of the Xenopus neural crest. Development, 130(3): 483-494.

Barnard RC, Pascall JC, Brown KD, McKay IA, Williams NS, Bustin SA. 1993. Coding sequence of ERF-1, the human homologue of Tis $11 \mathrm{~b} / \mathrm{cMG} 1$, members of the Tis 11 family of early response genes. Nucleic Acids Res, 21(15): 3580-3580.

Bell SE, Sanchez MJ, Spasic-Boskovic O, Santalucia T, Gambardella L, Burton GJ, Murphy JJ, Norton JD, Clark AR, Turner M. 2006. The

Kunming Institute of Zoology (CAS), China Zoological Society for cytoplasmic localization (Phillips et al, 2002). Our results indicated that the cytoplasmic localization of ZFP36L1 was important for its function, and suggested that ZFP36L1 was involved in neural development, likely by acting as an RNA-binding protein. However, the in vivo function of XZFP36L1 during brain development requires further detailed analysis.

Acknowledgements We thank the National Institute for Basic Biology, Japan, for the X1073b24 clone.
RNA binding protein $Z f p 36 l l$ is required for normal vascularisation and post-transcriptionally regulates VEGF expression. Dev Dyn, 235(11): 3144-3155.

Benjamin D, Schmidlin M, Min L, Gross B, Moroni C. 2006. BRF1 protein turnover and mRNA decay activity are regulated by protein kinase B at the same phosphorylation sites. Mol Cell Biol, 26(24): 9497-9507.

Blackshear PJ, Phillips RS, Ghosh S, Ramos SVB, Richfield EK, Lai WS. 2005. Zfp36l3, a rodent $\mathrm{X}$ chromosome gene encoding a placenta- 
specific member of the Tristetraprolin family of $\mathrm{CCCH}$ tandem zinc finger proteins. Biol Reprod, 73(2): 297-307.

Brennan SE, Kuwano Y, Alkharouf N, Blackshear PJ, Gorospe M, Wilson GM. 2009. The mRNA-destabilizing protein tristetraprolin is suppressed in many cancers, altering tumorigenic phenotypes and patient prognosis. Cancer Res, 69(12): 5168-5176.

Bowes JB, Snyder KA, Segerdell E, Gibb R, Jarabek C, Noumen E, Pollet N, Vize PD. 2008. Xenbase: a Xenopus biology and genomics resource. Nucleic Acids Res, 36(Database issue): D761-D767.

Carballo E, Lai WS, Blackshear PJ. 1998. Feedback inhibition of macrophage tumor necrosis factor- $\alpha$ production by tristetraprolin. Science, 281(5379): 1001-1005.

Carballo E, Lai WS, Blackshear PJ. 2000. Evidence that tristetraprolin is a physiological regulator of granulocyte-macrophage colonystimulating factor messenger RNA deadenylation and stability. Blood, 95(6): 1891-1899.

Carrick DM, Lai WS, Blackshear PJ. 2004. The tandem CCCH zinc finger protein tristetraprolin and its relevance to cytokine mRNA turnover and arthritis. Arthritis Res Ther, 6(6): 248-264.

Ciais D, Cherradi N, Bailly S, Grenier E, Berra E, Pouyssegur J, LaMarre J, Feige JJ. 2004. Destabilization of vascular endothelial growth factor mRNA by the zinc-finger protein Tis11b. Oncogene, 23(53): 8673-8680.

De J, Lai WS, Thorn JM, Goldsworthy SM, Liu X, Blackwell TK, Blackshear PJ. 1999. Identification of four $\mathrm{CCCH}$ zinc finger proteins in Xenopus, including a novel vertebrate protein with four zinc fingers and severely restricted expression. Gene, 228(1-2): 133-145.

Gawantka V, Delius H, Hirschfeld K, Blumenstock C, Niehrs C. 1995. Antagonizing the Spemann organizer: Role of the homeobox gene Xvent-1. EMBO J, 14(24): 6268-6279.

Hemmati-Brivanlou A, De La Torre J, Holt C, Harland R. 1991. Cephalic expression and molecular characterization of Xenopus En-2. Development, 111(3): 715-724.

Kaneko T, Chan TC, Satow R, Fujita T, Asashima M. 2003. The isolation and characterization of $\mathrm{XC} 3 \mathrm{H}-3 \mathrm{~b}$ : A $\mathrm{CCCH}$ zinc-finger protein required for pronephros development. Biochem Biophys Res Commun, 308(3): 566-572.

Kim P, Helms AW, Johnson JE, Zimmerman K. 1997. XATH-1, a vertebrate homolog of drosophila atonal, induces neuronal differentiation within ectodermal progenitors. Dev Biol, 187(1): 1-12.

Kurata T, Ueno N. 2003. Xenopus Nbx, a novel NK-1 related gene essential for neural crest formation. Dev Biol, 257(1): 30-40.

Lai WS, Blackshear PJ. 2001. Interactions of $\mathrm{CCCH}$ zinc-finger proteins with mRNA tristetraprolin-mediated AU-rich elementdependent mRNA degradation can occur in the absence of a poly(A) tail. J Biol Chem, 276(25): 23144-23154.

Lai WS, Stumpo D, Blackshear P. 1990. Rapid insulin-stimulated accumulation of an mRNA encoding a proline-rich protein. $J$ Biol Chem, 265(27): 16556-16563.

Lai WS, Carballo E, Thorn JM, Kennington EA, Blackshear PJ. 2000. Interactions of $\mathrm{CCCH}$ zinc finger proteins with mRNA. Binding of tristetraprolin-related zinc finger proteins to AU-rich elements and destabilization of mRNA. J Biol Chem, 275(23): 17827-17837.

Lai WS, Carballo E, Strum JR, Kennington EA, Phillips RS,
Blackshear PJ. 1999. Evidence that tristetraprolin binds to AU-rich elements and promotes the deadenylation and destabilization of tumor necrosis factor alpha mRNA. Mol Cell Biol, 19(6): 4311-43123.

Ma L, Zhao SH, Kong QH, Mao BY. 2007. Temporal and spatial expression patterns of Sox1 gene in Xenopus laevis embryo. Zool Res, 28(4): 403-408.

Ma PC, Zhao SH, Zeng WL, Yang QT, Li CC, Lv XY, Zhou Q, Mao BY. 2011. Xenopus Dbx2 is involved in primary neurogenesis and early neural plate patterning. Biochem Biophys Res Commun, 412(1): 170174.

Maitra S, Chou CF, Luber CA, Lee KY, Mann M, Chen CY. 2008. The AU-rich element mRNA decay-promoting activity of BRF1 is regulated by mitogen-activated protein kinase-activated protein kinase 2 . $R N A$, 14(5): 950-959.

Nieuwkoop PD, Faber J. 1967. Normal Table of Xenopus laevis (daudin) . New York and London: Garland Publishing.

Papalopulu N, Kintner C. 1996. A posteriorising factor, retinoic acid, reveals that anteroposterior patterning controls the timing of neuronal differentiation in Xenopus neuroectoderm. Development, 122(11): 3409-3418.

Phillips RS, Ramos SBV, Blackshear PJ. 2002. Members of the tristetraprolin family of tandem $\mathrm{CCCH}$ zinc finger proteins exhibit CRM1-dependent nucleocytoplasmic shuttling. J Biol Chem, 277(13): 11606-11613.

Ronquist F, Huelsenbeck JP. 2003. Mrbayes 3: Bayesian phylogenetic inference under mixed models. Bioinformatics, 19(12): 1572-1574.

Sato T, Sasai N, Sasai Y. 2005. Neural crest determination by coactivation of Pax 3 and Zicl genes in Xenopus ectoderm. Development, 132(10): 2355-2363.

Schmidlin M, Lu M, Leuenberger SA, Stoecklin G, Mallaun M, Gross B, Gherzi R, Hess D, Hemmings BA, Moroni C. 2004. The AREdependent mRNA-destabilizing activity of BRF1 is regulated by protein kinase B. EMBO J, 23(24): 4760-4769.

Sinha S, Dutta S, Datta K, Ghosh AK, Mukhopadhyay D. 2009. Von Hippel-Lindau gene product modulates TIS11B expression in renal cell carcinoma. J Biol Chem, 284(47): 32610-32618.

Stumpo DJ, Byrd NA, Phillips RS, Ghosh S, Maronpot RR, Castranio T, Meyers EN, Mishina Y, Blackshear PJ. 2004. Chorioallantoic fusion defects and embryonic lethality resulting from disruption of Zfp $36 L 1$, a gene encoding a $\mathrm{CCCH}$ tandem zinc finger protein of the Tristetraprolin family. Mol Cell Biol, 24(14): 6445-6455.

Thompson JD, Higgins DG, Gibson TJ. 1994. CLUSTAL W: Improving the sensitivity of progressive multiple sequence alignment through sequence weighting, position-specific gap penalties and weight matrix choice. Nucleic Acids Res, 22(22): 4673-4680.

Varnum BC, Ma QF, Chi TH, Fletcher B, Herschman HR. 1991. The TIS11 primary response gene is a member of a gene family that encodes proteins with a highly conserved sequence containing an unusual Cys-His repeat. Mol Cell Biol, 11(3): 1754-1758.

Wu JY, LI CC, Zhao SH, Mao BY. 2010. Differential expression of the Brunol/CELF family genes during Xenopus laevis early development. Int J Dev Biol, 54(1): 209-214.

Zhao SH, Jiang HF, Wang W, Mao BY. 2007. Cloning and developmental expression of the Xenopus Nkx6 genes. Dev Genes Evol, 217(6): 477-483. 